\title{
ARITHMETIC OF CHARACTER VARIETIES OF FREE GROUPS
}

\author{
SERGEY MOZGOVOY AND MARKUS REINEKE
}

\begin{abstract}
Via counting over finite fields, we derive explicit formulas for the $E$-polynomials and Euler characteristics of $\mathrm{GL}_{d^{-}}$and $\mathrm{PGL}_{d^{-}}$-character varieties of free groups. We prove a positivity property for these polynomials and relate them to the number of subgroups of finite index.
\end{abstract}

\section{INTRODUCTION AND STATEMENT OF THE RESULTS}

Given a finitely generated group $\Gamma$ and a complex reductive algebraic group $G$, one can consider the character variety

$$
X_{\Gamma}(G)=\operatorname{Hom}(\Gamma, G) / / G=\operatorname{Spec}\left(\mathbb{C}[\operatorname{Hom}(\Gamma, G)]^{G}\right),
$$

the spectrum of the (finitely generated) ring of $G$-invariant functions on the space of representations of $\Gamma$ in $G$. The study of these varieties, in particular for $\Gamma$ the fundamental group of a manifold, is a common theme in geometry and topology. Arithmetic aspects of character varieties have been studied very fruitfully e.g. in [7].

From now on let $\Gamma=F_{m}$ be the free group in $m \geq 0$ generators (i.e. the fundamental group of an $(m+1)$-punctured sphere). The character varieties $X_{\Gamma}(G)$ for the groups $G=\mathrm{GL}_{d}(\mathbb{C}), G=\mathrm{PGL}_{d}(\mathbb{C})$, and $G=\mathrm{SL}_{d}(\mathbb{C})$ were studied e.g. in 4. [5], and from an arithmetic point of view in [3]. We summarize some basic geometric properties of these representation varieties. For $m \geq 2$, the variety $X_{\Gamma}\left(\mathrm{GL}_{d}(\mathbb{C})\right)$ is an irreducible affine variety of dimension $(m-1) d^{2}+1$. It is usually a singular variety; its smooth locus typically reduces to $X_{\Gamma}^{\mathrm{irr}}\left(\mathrm{GL}_{d}(\mathbb{C})\right)$, the subset corresponding to irreducible representations.

Basic to the arithmetic study of character varieties is the following approach: there exists a $\mathbb{Z}$-model $\mathcal{X}_{d}$ of $X_{\Gamma}\left(\mathrm{GL}_{d}(\mathbb{C})\right)$ using Seshadri's GIT over base rings 15. Namely, consider the scheme

$$
\mathcal{X}_{d}=\operatorname{Spec}\left(\mathbb{Z}\left[\operatorname{Hom}\left(\Gamma, \mathrm{GL}_{d}(\mathbb{Z})\right)\right]^{\mathrm{GL}_{d}(\mathbb{Z})}\right)
$$

over $\operatorname{Spec}(\mathbb{Z})$. Then

$$
\operatorname{Spec}(\mathbb{C}) \times_{\operatorname{Spec}(\mathbb{Z})} \mathcal{X}_{d} \simeq X_{\Gamma}\left(\mathrm{GL}_{d}(\mathbb{C})\right)
$$

We find a similar open subscheme $\mathcal{X}_{d}^{\text {irr }} \subset \mathcal{X}_{d}$ of irreducible representations (over some open subscheme of $\operatorname{Spec}(\mathbb{Z}))$.

We can thus reduce to finite fields and consider the counting functions

$$
A_{d}(q)=\left|\mathcal{X}_{d}\left(\mathbb{F}_{q}\right)\right|, \quad A_{d}^{\mathrm{irr}}(q)=\left|\mathcal{X}_{d}^{\mathrm{irr}}\left(\mathbb{F}_{q}\right)\right|
$$

defined on prime powers $q$. By definition, $A_{d}(q)$ (resp. $A_{d}^{\text {irr }}(q)$ ) equals the number of isomorphism classes of completely reducible representations (resp. of absolutely irreducible representations, that is, representations that stay irreducible under any

Date: September 25, 2018. 
finite field extension) of the group $F_{m}$ of dimension $d$ over $\mathbb{F}_{q}$. Our first main result is the following:

Theorem 1.1. The varieties $X_{\Gamma}\left(\mathrm{GL}_{d}(\mathbb{C})\right)$ and $X_{\Gamma}^{\mathrm{irr}}\left(\mathrm{GL}_{d}(\mathbb{C})\right)$ are polynomial count 7. Appendix], that is, the functions $A_{d}(q)$ and $A_{d}^{\mathrm{irr}}(q)$ are polynomials with integer coefficients in $q$. Consequently, the E-polynomials of these varieties are

$$
E\left(X_{\Gamma}\left(\mathrm{GL}_{d}(\mathbb{C})\right) ; u, v\right)=A_{d}(u v), \quad E\left(X_{\Gamma}^{\mathrm{irr}}\left(\mathrm{GL}_{d}(\mathbb{C})\right) ; u, v\right)=A_{d}^{\mathrm{irr}}(u v) .
$$

Similar formulas for the group $\mathrm{PGL}_{d}(\mathbb{C})$ are proved in Corollary 2.6. The above theorem immediately follows from explicit formulas for $A_{d}(q)$ and $A_{d}^{\text {irr }}$ which we now formulate. Consider the formal power series ring $\mathbb{Q}(q) \llbracket t \rrbracket$ with the maximal ideal $\mathfrak{m}$. We define the $\mathbb{Q}(q)$-linear shift operator $T$ on $\mathbb{Q}(q) \llbracket t \rrbracket$ by

$$
T\left(t^{d}\right)=q^{(1-m)\left(\begin{array}{l}
d \\
2
\end{array}\right)} t^{d} .
$$

We define the plethystic exponential Exp : $\mathfrak{m} \rightarrow 1+\mathfrak{m}$ by

$$
\operatorname{Exp}\left(q^{i} t^{d}\right)=\left(1-q^{i} t^{d}\right)^{-1} \quad \text { and } \quad \operatorname{Exp}(f+g)=\operatorname{Exp}(f) \operatorname{Exp}(g) .
$$

It admits an inverse $\log : 1+\mathfrak{m} \rightarrow \mathfrak{m}$. Define the power operator 12 .

$$
\text { Pow }:(1+\mathfrak{m}) \times \mathbb{Q}(q) \llbracket t \rrbracket \rightarrow 1+\mathfrak{m}, \quad \operatorname{Pow}(f, g)=\operatorname{Exp}(g \log (f)) .
$$

Finally, define the series

$$
F(t)=\sum_{d \geq 0}\left((q-1) \ldots\left(q^{d}-1\right)\right)^{m-1} t^{d} \in \mathbb{Q}(q) \llbracket t \rrbracket .
$$

Theorem 1.2. In $\mathbb{Q}(q) \llbracket t \rrbracket$, we have

$$
\begin{aligned}
& \sum_{d \geq 0} A_{d}(q) t^{d}=\operatorname{Pow}\left(T^{-1} F(t)^{-1}, 1-q\right), \\
& \sum_{d \geq 1} A_{d}^{\mathrm{irr}}(q) t^{d}=(1-q) \log \left(T^{-1} F(t)^{-1}\right) .
\end{aligned}
$$

Using combinatorial methods, we prove a quite surprising positivity property of the counting polynomials $A_{d}(q)$ :

Theorem 1.3. We have $A_{d}(q) \in \mathbb{N}[q-1]$.

Contrarily, $A_{d}^{\mathrm{irr}}(q)$ does not fulfill this property. It is tempting to interpret this result geometrically, for example as a prediction for a paving of $X_{\Gamma}\left(\mathrm{GL}_{d}(\mathbb{C})\right.$ by tori (or at least quotients by finite groups of tori); but such a paving can not be expected to be very natural since it cannot be compatible with $X_{\Gamma}^{\mathrm{irr}}\left(\mathrm{GL}_{d}(\mathbb{C})\right)$. It would be interesting to test this prediction for the rather explicitly known $\mathrm{GL}_{2}(\mathbb{C})$-character varieties. We can determine explicitly the lowest order Taylor coefficient of $A_{d}(q)$ around $q=1$ to derive:

Theorem 1.4. For $m \geq 2$, the topological Euler characteristic of the varieties $X_{\Gamma}\left(\mathrm{PGL}_{d}(\mathbb{C})\right)$ and $X_{\Gamma}^{\mathrm{irr}}\left(\mathrm{PGL}_{d}(\mathbb{C})\right)$ are given by

$$
\begin{gathered}
\chi\left(X_{\Gamma}\left(\mathrm{PGL}_{d}(\mathbb{C})\right)\right)=\varphi(d) d^{m-2}, \\
\chi\left(X_{\Gamma}^{\mathrm{irr}}\left(\mathrm{PGL}_{d}(\mathbb{C})\right)\right)=\mu(d) d^{m-2},
\end{gathered}
$$

where $\varphi$ and $\mu$ denote the Euler totient function, resp. the Möbius function. 
Again, it would be interesting to have a geometrical explanation for these Euler characteristics. We can also count absolutely indecomposable representations (that is, representations that stay indecomposable under any finite field extension) of $F_{m}$ over finite fields:

Theorem 1.5. For any $d \geq 1$, there exists a polynomial $A_{d}^{\text {ind }}(q) \in \mathbb{Z}[q]$ that counts isomorphism classes of absolutely indecomposable representations of $F_{m}$ of dimension $d$ over $\mathbb{F}_{q}$ for every prime power $q$. These polynomials satisfy

$$
\sum_{d \geq 1} A_{d}^{\mathrm{ind}}(q) t^{d}=(q-1) \log \left(\sum_{\lambda} r_{\lambda}^{m-1} t^{|\lambda|}\right),
$$

where the sum ranges over all partitions and, for any partition $\lambda$,

$$
r_{\lambda}=\prod_{n \geq 1} q^{\lambda_{n}^{2}}\left(q^{-1}\right)_{\lambda_{n}-\lambda_{n+1}}, \quad(q)_{n}=(1-q) \ldots\left(1-q^{n}\right) .
$$

In contrast to [3], our approach to the arithmetic of the character varieties is non-geometric and purely formal. It is based on the Hall algebra approach to the arithmetic of moduli spaces of representations of quivers developed in [14. In fact, we can directly adopt the methods there to derive the explicit formula for $A_{d}(q)$. The other results follow by a detailed study of the right hand side of this formula and explicit combinatorics. One can expect this approach to even work in a motivic Hall algebra [1, leading to a formula similar to Theorem 1.2 for the motives of character varieties.

The paper is organized as follows: in Section 2, we recall the Hall algebra methods of 14 to prove Theorems 1.1 and 1.2. In Section 3. we derive Theorem 1.4 using an elementary argument. Combinatorial notions are introduced in Section 4 to derive Theorem 1.3. In Section 5, we derive a formula for the number of absolutely indecomposable representations of $F_{m}$ over finite fields. In Section 6, we explain how the numbers of subgroups of fixed index in free groups [6] can be reconstructed from the counting polynomials, providing a potential link of the present study with the topic of subgroup growth [10].

\section{Computation of the COUnting POlynomial}

Let $\Gamma=F_{m}$ be the free group in $m$ generators and let $k$ be a field. A representation of the group algebra $k \Gamma$ can be identified with a representation of the free associative algebra $A$ with $m$ generators such that all generators act bijectively on the representation. Therefore the category of $k \Gamma$-representations can be identified with an exact subcategory of the abelian category of $A$-representations. Since the algebra $A$ is a path algebra of the quiver with one vertex and $m$ loops, the methods of [14] for the explicit calculation of the number of isomorphism classes of absolutely irreducible representations of quivers continue to work for $\Gamma$-representations. We recall the main steps of this calculation and refer to [14 for the proofs which hold without any changes.

In the following, let $k$ be a finite field with $q$ elements. We first define the Hall algebra $H((k \Gamma))$ of the group algebra $k \Gamma$. As a (complete, $\mathbb{Z}_{\geq 0}$-graded) $\mathbb{Q}$-vector space, we define

$$
H((k \Gamma))=\prod_{[V]} \mathbb{Q} \cdot[V]
$$


where the direct product ranges over all isomorphism classes of (finite-dimensional) representations $V$ of $k \Gamma$. We have a natural grading by the $\operatorname{dimension} \operatorname{dim} V$. We define a product on $H((k \Gamma))$ by

$$
[V] \cdot[W]=\sum_{[X]} g_{V, W}^{X}[X]
$$

where $g_{V, W}^{X}$ equals the number of subrepresentations $U \subset X$ such that $U$ is isomorphic to $W$ and $X / U$ is isomorphic to $V$. Then we have [14,3.3]:

Lemma 2.1. This product endows $H((k \Gamma))$ with a structure of a $\mathbb{Z}_{\geq 0}$-graded complete local associative unital $\mathbb{Q}$-algebra. In particular, every element with constant term 1 (the class of the zero-dimensional representation) is invertible in $H((k \Gamma))$.

We define an evaluation map

$$
I: H((k \Gamma)) \rightarrow \mathbb{Q} \llbracket t \rrbracket, \quad[V] \mapsto \frac{1}{|\operatorname{Aut}(V)|} t^{\operatorname{dim} V} .
$$

By [14, Lemma 3.4], we have:

Lemma 2.2. The composition $T \circ I: H((k \Gamma)) \rightarrow \mathbb{Q} \llbracket t \rrbracket$ is a $\mathbb{Q}$-algebra homomorphism, where the operator $T$ is defined by (11).

We consider the (invertible) element

$$
e=\sum_{[V]}[V] \in H((k \Gamma)) .
$$

Using $\operatorname{Hom}\left(\Gamma, \mathrm{GL}_{d}(k)\right) \simeq \mathrm{GL}_{d}(k)^{m}$ and

$$
\left|\mathrm{GL}_{d}(k)\right|=\prod_{i=0}^{d-1}\left(q^{d}-q^{i}\right)=q^{\left(\begin{array}{c}
d \\
2
\end{array}\right)} \prod_{i=1}^{d}\left(q^{i}-1\right)
$$

the following is easily verified:

Lemma 2.3. We have

$$
I(e)=T^{-1} F(t), \quad I\left(e^{-1}\right)=T^{-1} F(t)^{-1},
$$

where $F(t)$ is defined by (3).

Proof. By the definitions, we have

$$
I(e)=\sum_{d \geq 0} \frac{\left|\mathrm{GL}_{d}(k)^{m}\right|}{\left|\mathrm{GL}_{d}(k)\right|} t^{d}=\sum_{d \geq 0}\left(q^{\left(\begin{array}{c}
d \\
2
\end{array}\right)} \prod_{i=1}^{d}\left(q^{i}-1\right)\right)^{m-1} t^{d}=T^{-1} F(t) .
$$

This implies $T I(e)=F(t)$ and therefore $T I\left(e^{-1}\right)=F(t)^{-1}$ by Lemma 2.2. Therefore $I\left(e^{-1}\right)=T^{-1} F(t)^{-1}$.

The key lemma towards counting absolutely irreducible representations is [14, Lemma 3.5]:

Lemma 2.4. Writing

$$
e^{-1}=\sum_{[V]} \gamma_{V}[V]
$$

in $H((k \Gamma))$, we have the following description of the coefficients $\gamma_{V}$

(1) If $V$ is not completely reducible, then $\gamma_{V}=0$. 
(2) If $V=\bigoplus_{[S]} S^{m_{S}}$ is completely reducible (the direct sum ranging over all isomorphism classes of irreducible representations $S$ of $k \Gamma$ ), then

$$
\gamma_{V}=\prod_{[S]}(-1)^{m_{S}}|\operatorname{End}(S)|^{\left(\begin{array}{c}
m_{S} \\
2
\end{array}\right)} .
$$

Based on this lemma, one can prove (see [14, Theorem 4.2]) that:

Theorem 2.5. The function $A_{d}^{\mathrm{irr}}(q)$ is a polynomial in $q$ and we have in $\mathbb{Q} \llbracket t \rrbracket$

$$
I\left(e^{-1}\right)=\operatorname{Exp}\left(\frac{1}{1-q} \sum_{d \geq 1} A_{d}^{\mathrm{irr}}(q) t^{d}\right)_{q=|k|} .
$$

Proof of Theorems 1.1 and 1.2. We obtain from Theorem 2.5 and Lemma 2.3 that $A_{d}^{\text {irr }}(q)$ is a polynomial in $q$ satisfying in $\mathbb{Q}(q) \llbracket t \rrbracket$

$$
\sum_{d \geq 1} A_{d}^{\mathrm{irr}}(q) t^{d}=(1-q) \log \left(T^{-1} F(t)^{-1}\right) .
$$

This implies that $A_{d}^{\mathrm{irr}}(q)$ has integer coefficients. Using [12, Lemma 5], one can prove that $A_{d}(q)$ is a polynomial in $q$ satisfying in $\mathbb{Q}(q) \llbracket t \rrbracket$

$$
\sum_{d \geq 0} A_{d}(q) t^{d}=\operatorname{Exp}\left(\sum_{d \geq 1} A_{d}^{\mathrm{irr}}(q) t^{d}\right) .
$$

Therefore

$$
\sum_{d \geq 0} A_{d}(q) t^{d}=\operatorname{Exp}\left((1-q) \log \left(T^{-1} F(t)^{-1}\right)\right)=\operatorname{Pow}\left(T^{-1} F(t)^{-1}, 1-q\right)
$$

and $A_{d}(q)$ has integer coefficients. This finishes the proof of Theorem 1.2, To prove Theorem [1.1 we note that by [7, Appendix], the E-polynomials are given by the counting polynomials evaluated at $q=u v$.

Now we pass to the $\mathrm{PGL}_{d}(\mathbb{C})$-character varieties. There is a free action of the torus $\left(\mathbb{C}^{*}\right)^{m}$ on $X_{\Gamma}\left(\mathrm{GL}_{d}(\mathbb{C})\right)$. The quotient fibration $X_{\Gamma}\left(\mathrm{GL}_{d}(\mathbb{C})\right) \rightarrow X_{\Gamma}\left(\mathrm{PGL}_{d}(\mathbb{C})\right)$ has fibres isomorphic to $\left(\mathbb{C}^{*}\right)^{m}$ and is Zariski locally trivial by Hilbert 90 . Similarly for the open subsets corresponding to irreducible representations.

Consequently, we see that the $E$-polynomials of the $\mathrm{PGL}_{d}(\mathbb{C})$-character varieties are known:

Corollary 2.6. We have

$$
\begin{gathered}
E\left(X_{\Gamma}\left(\mathrm{PGL}_{d}(\mathbb{C})\right) ; u, v\right)=\frac{1}{(u v-1)^{m}} A_{d}(u v), \\
E\left(X_{\Gamma}\left(\mathrm{PGL}_{d}(\mathbb{C})\right)^{\mathrm{irr}} ; u, v\right)=\frac{1}{(u v-1)^{m}} A_{d}^{\mathrm{irr}}(u v) .
\end{gathered}
$$

We conclude this section with an example. First, we have

$$
A_{1}^{\mathrm{irr}}(q)=A_{1}(q)=(q-1)^{m} \text {. }
$$

As the first nontrivial special case of Theorem 1.2, we have $A_{2}^{\mathrm{irr}}(q)=$

$$
(q-1)^{m}\left(q^{m-1}(q-1)^{m-1}\left((q+1)^{m-1}-1\right)-\frac{1}{2}(q+1)^{m-1}+\frac{1}{2}(q-1)^{m-1}\right) .
$$


This implies

$$
\begin{aligned}
& A_{2}(q)=A_{2}^{\mathrm{irr}}(q)+\frac{1}{2}\left(A_{1}^{\mathrm{irr}}\left(q^{2}\right)+A_{1}^{\mathrm{irr}}(q)^{2}\right)= \\
& (q-1)^{m}\left(q^{m-1}(q-1)^{m-1}\left((q+1)^{m-1}-1\right)+\frac{1}{2} q\left((q+1)^{m-1}+(q-1)^{m-1}\right)\right)
\end{aligned}
$$

and thus

$$
\begin{aligned}
& E\left(X_{\Gamma}\left(\mathrm{PGL}_{2}(\mathbb{C})\right) ; u, v\right)=\frac{1}{(u v-1)^{m}} A_{2}(u v) \\
= & (u v)^{m-1}(u v-1)^{m-1}\left((u v+1)^{m-1}-1\right)+\frac{1}{2} u v\left((u v+1)^{m-1}+(u v-1)^{m-1}\right),
\end{aligned}
$$

which should be compared with $[\underline{3}$, Theorem B].

\section{EULER CHARACTERISTIC}

In this section, we prove Theorem 1.4 We first need a lemma on the behaviour of specialization at $q=1$ for special plethystic exponentials and logarithms. Let $A$ be the subring of $\mathbb{Q}(q)$ consisting of all rational functions without pole at $q=1$.

Lemma 3.1. Assume that

$$
\log \left(1+(q-1)^{m} \sum_{n \geq 1} a_{n}(q) t^{n}\right)=(q-1)^{m} \sum_{n \geq 1} b_{n}(q) t^{n} \in \mathbb{Q}(q) \llbracket t \rrbracket .
$$

Then $a_{n}(q) \in A$ for all $n \geq 1$ if and only if $b_{n}(q) \in A$ for all $n \geq 1$. Moreover,

$$
b_{n}(1)=\sum_{d \mid n} a_{n / d}(1) \mu(d) d^{m-1}, \quad a_{n}(1)=\sum_{d \mid n} b_{n / d}(1) d^{m-1} .
$$

Proof. We recall a more direct definition of the operators Exp and Log on formal power series: for $n \geq 1$, define the $\mathbb{Q}$-linear Adams operator $\psi_{n}$ on $R=\mathbb{Q}(q) \llbracket t \rrbracket$ by $\psi_{n}\left(q^{i} t^{j}\right)=q^{n i} t^{n j}$, and define $\Psi=\sum_{n \geq 1} \frac{1}{n} \psi_{n}$ with inverse $\Psi^{-1}=\sum_{n \geq 1} \frac{\mu(n)}{n} \psi_{n}$. Then $\operatorname{Exp}=\exp \circ \Psi$ and $\log =\Psi^{-1} \circ \log$.

Using this description,we immediately derive the following formula:

$$
\log \left(1+\sum_{n \geq 1} r_{n}(q) t^{n}\right)=\sum_{n=1}^{\infty} \sum_{i j=n} \frac{\mu(i)}{i} \sum_{j=c_{1}+\ldots+c_{l}} \frac{(-1)^{l-1}}{l} \prod_{k=1}^{l} r_{c_{k}}\left(q^{i}\right) t^{n} .
$$

Now if $r_{n}(q)=(q-1)^{m} a_{n}(q)$, then

$$
\begin{aligned}
\sum_{n \geq 1} b_{n}(q) t^{n}=(q-1)^{-m} \log \left(1+(q-1)^{m} \sum_{n \geq 1} a_{n}(q) t^{n}\right) \\
=\sum_{n=1}^{\infty} \sum_{i j=n} \frac{\mu(i)}{i} \sum_{j=c_{1}+\ldots+c_{l}} \frac{(-1)^{l-1}}{l} \frac{\left(q^{i}-1\right)^{m l}}{(q-1)^{m}} \prod_{k=1}^{l} a_{c_{k}}\left(q^{i}\right) t^{n} .
\end{aligned}
$$

We see that the summand corresponding to the decomposition $j=c_{1}+\cdots+c_{l}$ has a zero of order at least $m(l-1)$ at $q=1$. Specializing this formula at $q=1$, 
we see that only terms with $l=1$ (and thus $c_{1}=j$ ) contribute. Thus the formula simplifies to

$$
b_{n}(1)=\sum_{i j=n} \frac{\mu(i)}{i} i^{m} a_{j}(1)=\sum_{i j=n} a_{j}(1) \mu(i) i^{m-1},
$$

and the first claim follows.

Similarly, we prove the second claim. Using the above description of Exp we derive the following formula:

$$
\operatorname{Exp}\left(\sum_{n \geq 1} r_{n}(q) t^{n}\right)=1+\sum_{n=1}^{\infty} \sum_{n=c_{1}+\ldots+c_{l}} \frac{1}{l !} \sum_{\left(i_{k} j_{k}=c_{k}\right)_{k}} \prod_{k=1}^{l} \frac{r_{j_{k}}\left(q^{i_{k}}\right)}{i_{k}} t^{n} .
$$

Now if $r_{n}(q)=(q-1)^{m} b_{n}(q)$, this formula yields

$$
\begin{aligned}
\sum_{n \geq 1} a_{n}(q) t^{n}= & \frac{1}{(q-1)^{m}}\left(\operatorname{Exp}\left((q-1)^{m} \sum_{n \geq 1} b_{n}(q) t^{n}\right)-1\right) \\
& =\sum_{n=1}^{\infty} \sum_{n=c_{1}+\ldots+c_{l}} \frac{1}{l !} \sum_{\left(i_{k} j_{k}=c_{k}\right)_{k}} \prod_{k=1}^{l} \frac{b_{j_{k}}\left(q^{i_{k}}\right)\left(q^{i_{k}}-1\right)^{m}}{i_{k}} \frac{1}{(q-1)^{m}} t^{n} .
\end{aligned}
$$

As soon as $l \geq 2$ in a summand on the right hand side, this summand specializes to zero at $q=1$. Thus, after this specialization, we only have to consider summands with $l=1$, and thus $c_{1}=n$, which reads

$$
a_{n}(1)=\left.\sum_{i j=n} \frac{b_{j}(1)}{i} \frac{\left(q^{i}-1\right)^{m}}{(q-1)^{m}}\right|_{q=1}=\sum_{i j=n} b_{j}(1) i^{m-1},
$$

as claimed.

We can now prove Theorem 1.4:

Proof. We can write the series $T^{-1} F(t)^{-1}$ of the previous sections in the form

$$
T^{-1} F(t)^{-1}=1+\sum_{d \geq 1}(q-1)^{d(m-1)} a_{d}(q) t^{d}
$$

for some $a_{d}(q) \in \mathbb{Q}[q]$ with $a_{1}(q)=-1$. By Theorem 1.2 , we have

$$
\frac{1}{(q-1)^{m}} \sum_{d \geq 1} A_{d}^{\mathrm{irr}}(q) t^{d}=-\frac{1}{(q-1)^{m-1}} \log \left(1+\sum_{d \geq 1}(q-1)^{d(m-1)} a_{d}(q) t^{d}\right)
$$

and applying Lemma 3.1, we obtain

$$
\left.\frac{1}{(q-1)^{m}} A_{d}^{\mathrm{irr}}(q)\right|_{q=1}=-a_{1}(1) \mu(d) d^{m-2}=\mu(d) d^{m-2},
$$

which, together with Corollary 2.6. proves the first part of the theorem. Setting $b_{d}(q)=(q-1)^{-m} A_{d}^{\mathrm{irr}}(q)$ we can write, by Theorem 1.2 .

$$
\sum A_{d}(q) t^{d}=\operatorname{Exp}\left((q-1)^{m} \sum_{d \geq 1} b_{d}(q) t^{d}\right)
$$


and applying Lemma 3.1 again, we derive

$$
\begin{aligned}
\left.\frac{1}{(q-1)^{m}} A_{d}(q)\right|_{q=1}=\sum_{i j=d} b_{j}(1) i^{m-1}=\sum_{i j=d} \mu & (j) j^{m-2} i^{m-1} \\
& =\sum_{i j=d} \mu(j) i \cdot d^{m-2}=\varphi(d) d^{m-2}
\end{aligned}
$$

proving the second part of the theorem, again by Corollary 2.6.

\section{Positivity}

The goal of this section is to prove Theorem 1.3, that is, that the polynomials $A_{d}(q)$ determined in the previous sections satisfy

$$
A_{d} \in \mathbb{N}[s], \quad s=q-1 .
$$

By Theorem [1.2, we have

$$
\sum_{d \geq 0} A_{d} t^{d}=\operatorname{Pow}\left(T^{-1} F(t)^{-1}, 1-q\right),
$$

where the series $F(t)$, defined in (3), can be written in the form

$$
F(t)=\sum_{d \geq 0}[d]_{q}^{!(m-1)}\left((q-1)^{m-1} t\right)^{d}
$$

with $[d]_{q}^{!}=\prod_{i=1}^{d} \frac{q^{i}-1}{q-1}$. We will prove positivity in several steps.

\subsection{Positivity of the inverse. Let}

$$
F(t)^{-1}=1-\sum_{n \geq 1} a_{n}(q) t^{n}
$$

We claim that $a_{n} \in \mathbb{N}[s]$, where $s=q-1$. We will prove actually a stronger result.

Let $S_{n}$ be the group of permutations of $[n]=\{1, \ldots, n\}$. For any $\sigma \in S_{n}$, let $l(\sigma)$ denote its length. It can be described as a number of inversions

$$
l(\sigma)=|\{i<j \mid \sigma(i)>\sigma(j)\}| .
$$

Let $G_{n}=S_{n}^{m-1}$ and, for any $\sigma=\left(\sigma_{1}, \ldots, \sigma_{m-1}\right) \in G_{n}$, let

$$
l(\sigma)=l\left(\sigma_{1}\right)+\cdots+l\left(\sigma_{m-1}\right) .
$$

For any $n \geq 1$, let $P_{n} \subset G_{n}$ be the set of connected elements, that is, elements $\sigma=\left(\sigma_{1}, \ldots, \sigma_{m-1}\right)$ such that there is no subinterval $[k]$ for $k<n$, fixed by all $\sigma_{i}$.

Theorem 4.1. Let

$$
\left(\sum_{n \geq 0}[n]_{q}^{!(m-1)} t^{n}\right)^{-1}=1-\sum_{n \geq 1} a_{n} t^{n} .
$$

Then

$$
a_{n}=\sum_{\sigma \in P_{n}} q^{l(\sigma)} \in \mathbb{N}[q]
$$


Proof. It is known [11, Ch. III, Eq. 1.3.viii] that

$$
\sum_{\sigma \in S_{n}} q^{l(\sigma)}=[n]_{q}^{!} .
$$

This implies that

$$
\sum_{n \geq 0}[n]_{q}^{(m-1)} t^{n}=\sum_{n \geq 0} \sum_{\sigma \in G_{n}} q^{l(\sigma)} t^{n} .
$$

The theorem will be proved if we will show that

$$
\left(\sum_{n \geq 0} \sum_{\sigma \in G_{n}} q^{l(\sigma)} t^{n}\right)\left(1-\sum_{n \geq 1} \sum_{\sigma \in P_{n}} q^{l(\sigma)} t^{n}\right)=1
$$

or equivalently

$$
\sum_{\sigma \in G_{n}} q^{l(\sigma)}=\sum_{k+l=n, l \geq 1} \sum_{\sigma \in G_{k}} \sum_{\tau \in P_{l}} q^{l(\sigma)+l(\tau)} .
$$

Any element in $G_{n}$ can be uniquely written in the form $(\sigma, \tau) \in G_{k} \times P_{l}$ where $k+l=n, k \geq 0$, and $l \geq 1$. It is clear that the length of $(\sigma, \tau)$ is equal to $l(\sigma)+l(\tau)$ and the theorem follows.

Applying this theorem to (6), we see that the polynomials $a_{n}$ determined by (7) are contained in $\mathbb{N}[s]$. The same is then true if we substitute $F(t)^{-1}$ by $T^{-1} F(t)^{-1}$.

4.2. Positivity of the power. The goal of this section is to prove the following result

Theorem 4.2. Let

$$
f=1-\sum_{n \geq 1} a_{n}(q) t^{n} \in \mathbb{Q}[q] \llbracket t \rrbracket,
$$

where $a_{n} \in \mathbb{Q}_{>0}[q-1]$. Then

$$
\operatorname{Pow}(f, 1-q) \in \mathbb{Q}_{\geq 0}[q-1] \llbracket t \rrbracket .
$$

Applying this result to $f=T^{-1} F(t)^{-1}$, we prove (4) and therefore prove Theorem 1.3. In order to prove the above theorem we will use the formula for Pow $(f, 1-$ q) proved in [12, Lemma 22]. Let $\Phi_{d}(q)$ be the number of irreducible monic polynomials of degree $d$ over $\mathbb{F}_{q}$ with a nonzero constant coefficient . Then

$$
\begin{gathered}
\psi_{n}(q-1)=q^{n}-1=\sum_{d \mid n} d \Phi_{d}(q), \\
\Phi_{n}(q)=\frac{1}{n} \sum_{d \mid n} \mu(n / d)\left(q^{d}-1\right)=\frac{1}{n} \sum_{d \mid n} \mu(n / d) q^{d}-\delta_{n 1} .
\end{gathered}
$$

It is proved in [12, Lemma 22] that

$$
\operatorname{Pow}(f, 1-q)=\prod_{d \geq 1} \psi_{d}(f)^{-\Phi_{d}},
$$

where on the right we use $f^{g}=\operatorname{pow}(f, g)=\exp (g \log (f))$. We will show that for $f$ as in the theorem, each multiple on the right is in $\mathbb{Q} \geq 0[s] \llbracket t \rrbracket$, where $s=q-1$, and therefore $\operatorname{Pow}(f, 1-q) \in \mathbb{Q}_{\geq 0}[s] \llbracket t \rrbracket$ as required.

Remark 4.3. Note that $\psi_{n}(s)=(s+1)^{n}-1 \in \mathbb{N}[s]$. Therefore, if $f \in \mathbb{Q}_{\geq 0}[s]$, then $\psi_{n}(f) \in \mathbb{Q}_{\geq 0}[s]$. 
Remark 4.4. Computer tests show that if $f \in \mathbb{Q}_{\geq 0}[s] \llbracket t \rrbracket$, then $\operatorname{Pow}(f, q-1) \in$ $\mathbb{Q}_{\geq 0}[s] \llbracket t \rrbracket$. This is slightly different from our statement. Our strategy of the proof will not work in this case as the multiples on the right of the form $\operatorname{pow}\left(f, s^{k}\right)$ are not in $\mathbb{Q}_{\geq 0}[s] \llbracket t \rrbracket$ in general.

Lemma 4.5. For any $n \geq 1$, we have

$$
n \Phi_{n}(q) \in \mathbb{N}[q-1] .
$$

Proof. We will use the idea from [2]. For $n \geq 2$, we have

$$
n \Phi_{n}(s+1)=\sum_{d \mid n} \mu(d)(s+1)^{n / d}=\sum_{k \geq 0} \sum_{d \mid n} \mu(d)\left(\begin{array}{c}
n / d \\
k
\end{array}\right) s^{k} .
$$

For any $d \mid n$, we have

$$
\begin{aligned}
\left(\begin{array}{c}
n / d \\
k
\end{array}\right)=\mid\left\{1 \leq a_{1}<\cdots<a_{k}\right. & \leq n / d\} \mid \\
& =\left|\left\{1 \leq a_{1}<\cdots<a_{k} \leq n|d| \operatorname{gcd}\left(a_{1}, \ldots, a_{k}\right)\right\}\right| .
\end{aligned}
$$

Therefore

$$
\begin{array}{r}
\sum_{d \mid n} \mu(d)\left(\begin{array}{c}
n / d \\
k
\end{array}\right)=\sum_{d \geq 1} \mu(d) \cdot\left|\left\{1 \leq a_{1}<\cdots<a_{k} \leq n|d| \operatorname{gcd}\left(a_{1}, \ldots, a_{k}, n\right)\right\}\right| \\
=\left|\left\{1 \leq a_{1}<\cdots<a_{k} \leq n \mid \operatorname{gcd}\left(a_{1}, \ldots, a_{k}, n\right)=1\right\}\right| .
\end{array}
$$

Indeed, for any tuple $1 \leq a_{1}<\cdots<a_{k} \leq n$ with $\operatorname{gcd}\left(a_{1}, \ldots, a_{k}\right)=m$, its contribution to the second sum is

$$
\sum_{d \mid \operatorname{gcd}(m, n)} \mu(d)=\delta_{\operatorname{gcd}(m, n), 1}
$$

Lemma 4.6. Let

$$
f=1-\sum_{n \geq 1} a_{n}(q) t^{n} \in \mathbb{Q}(q) \llbracket t \rrbracket,
$$

where $a_{n} \in \mathbb{Q}_{\geq 0}[s]$ and let $g \in \mathbb{Q}_{\geq 0}[s]$. Then

$$
\operatorname{pow}(f,-g) \in \mathbb{Q}_{\geq 0}[s] \llbracket t \rrbracket .
$$

Proof. It is enough to show that pow $(1-t,-g) \in \mathbb{Q}_{\geq 0}[s] \llbracket t \rrbracket$. But

$$
\operatorname{pow}(1-t,-g)=\exp (-g \log (1-t))=\exp \left(g \sum_{n \geq 1} \frac{t^{n}}{n}\right) \in \mathbb{Q}_{\geq 0}[s] \llbracket t \rrbracket .
$$

Applying this lemma to $\psi_{d}(f)^{-\Phi_{d}}$ for $d \geq 1$ and using Formula (8), we prove Theorem 4.2 . 


\section{Counting indecomposable REPRESENTAtions}

Let $k$ be a finite field. For any $d \geq 0$, let $G_{d}=\mathrm{GL}_{d}(k)$ and $R_{d}=\operatorname{Hom}\left(\Gamma_{m}, G_{d}\right)$. Then $G_{d}$ acts on $R_{d}$ by conjugation. Using the Kac-Stanley-Hua approach [8, 12, we will prove a formula for the number of $G_{d}$-orbits in $R_{d}$ :

Theorem 5.1. We have

$$
\sum_{d \geq 0}\left|R_{d} / G_{d}\right| t^{d}=\operatorname{Pow}\left(\sum_{\lambda} r_{\lambda}(q)^{m-1} t^{|\lambda|}, q-1\right)_{q=|k|}
$$

where the sum ranges over all partitions and, for any partition $\lambda$,

$$
r_{\lambda}(q)=\prod_{n \geq 1} q^{\lambda_{n}^{2}}\left(q^{-1}\right)_{\lambda_{n}-\lambda_{n+1}}, \quad(q)_{n}=(1-q) \ldots\left(1-q^{n}\right) .
$$

Proof. Using the Burnside formula, we can write

$$
\left|R_{d} / G_{d}\right|=\sum_{[g] \in G_{d} / \sim}\left|R_{d}^{g}\right| /\left|G_{d}^{g}\right|=\sum_{g \in G_{d} / \sim}\left|G_{d}^{g}\right|^{m-1}
$$

where the sum ranges over the conjugacy classes, $R_{d}^{g}$ is the set of $g$-invariant elements in $R_{d}$, and $G_{d}^{g}$ is the centralizer of $g$.

Let us describe the set of conjugacy classes of $G_{d}$. Let $\Phi$ be the set of all monic irreducible polynomials in a variable $t$ over $k$ with a nonzero constant coefficient. The Jordan blocks of invertible matrices are parametrized by pairs $(n, f)$, where $n \geq 1$ and $f \in \Phi$. The Jordan block $J(n, f)$ corresponds to the action of $x$ on $k[x] /\left(f^{n}\right)$ and has size $n \cdot \operatorname{deg} f$. Conjugacy classes in $G_{d}$ are parametrized by maps

$$
\varphi: \mathbb{N}_{>0} \times \Phi \rightarrow \mathbb{N}
$$

such that $\sum_{f \in \Phi} \operatorname{deg} f \sum_{n \geq 1} n \varphi(n, f)=d$. Equivalently, we can consider $\varphi$ as a map $\Phi \rightarrow \mathcal{P}$, where $\mathcal{P}=\operatorname{Map}_{0}\left(\mathbb{N}_{>0}, \mathbb{N}\right)$ is the set of maps with finite support. The set $\mathcal{P}$ can be identified with the set of all partitions, where to any $m \in \mathcal{P}$ we associate the usual partition $\lambda$ with $\lambda_{n}=\sum_{i \geq n} m_{i}$. Note that $|\lambda|=\sum_{n \geq 1} \lambda_{n}=\sum_{n \geq 1} n m_{n}$.

The conjugacy class corresponding to $\varphi: \Phi \rightarrow \mathcal{P}$ is given by the action of $x$ on

$$
V_{\varphi}=\bigoplus_{f \in \Phi} \bigoplus_{n \geq 1}\left(k[x] /\left(f^{n}\right)\right)^{\varphi(n, f)} .
$$

Its centralizer has order [13, Theorem 2.1]

$$
\left|\operatorname{Aut}\left(V_{\varphi}\right)\right|=\left|\operatorname{End}\left(V_{\varphi}\right)\right| \prod_{f \in \Phi} \prod_{n \geq 1}\left(q^{-\operatorname{deg} f}\right)_{\varphi(n, f)}
$$

where $q=|k|$,

$$
\operatorname{dim} \operatorname{End}\left(V_{\varphi}\right)=\sum_{f \in \Phi} \operatorname{deg} f \sum_{k, l \geq 1} \min \{k, l\} \varphi(k, f) \varphi(l, f)=\sum_{f \in \Phi} \operatorname{deg} f \sum_{n \geq 1} \lambda(f)_{n}^{2}
$$

and $\lambda(f)$ is the partition corresponding to $\varphi(-, f) \in \mathcal{P}$. This implies

$$
\begin{aligned}
\left|\operatorname{Aut}\left(V_{\varphi}\right)\right| & =\prod_{f \in \Phi} \prod_{n \geq 1} q^{\operatorname{deg} f \cdot \lambda(f)_{n}^{2}}\left(q^{-\operatorname{deg} f}\right)_{\varphi(n, f)} \\
& =\prod_{f \in \Phi} \psi_{\operatorname{deg} f}\left(\prod_{n \geq 1} q^{\lambda(f)_{n}^{2}}\left(q^{-1}\right)_{\lambda(f)_{n}-\lambda(f)_{n+1}}\right)=\prod_{f \in \Phi} \psi_{\operatorname{deg} f}\left(r_{\lambda(f)}(q)\right),
\end{aligned}
$$


where $\psi_{n}$ is the Adams operation. Therefore, applying (9), we obtain

$$
\left|R_{d} / G_{d}\right|=\sum_{\substack{\lambda: \Phi \rightarrow \mathcal{P} \\ \sum_{f} \operatorname{deg} f \cdot|\lambda(f)|=d}} \prod_{f \in \Phi} \psi_{\operatorname{deg} f}\left(r_{\lambda(f)}(q)^{m-1}\right) .
$$

This implies

$$
\begin{aligned}
& \sum_{d \geq 1}\left|R_{d} / G_{d}\right| t^{d}=\sum_{\lambda: \Phi \rightarrow \mathcal{P}}\left(\prod_{f \in \Phi} \psi_{\operatorname{deg} f}\left(r_{\lambda(f)}(q)^{m-1}\right)\right) t^{\sum_{f \in \Phi} \operatorname{deg} f \cdot|\lambda(f)|} \\
&=\sum_{\lambda: \Phi \rightarrow \mathcal{P}} \prod_{f \in \Phi} \psi_{\operatorname{deg} f}\left(r_{\lambda(f)}(q)^{m-1} t^{|\lambda(f)|}\right)=\prod_{f \in \Phi} \psi_{\operatorname{deg} f}\left(\sum_{\lambda \in \mathcal{P}} r_{\lambda}(q)^{m-1} t^{|\lambda|}\right) \\
&=\prod_{d \geq 1} \psi_{d}\left(\sum_{\lambda \in \mathcal{P}} r_{\lambda}(q)^{m-1} t^{|\lambda|}\right)^{\Phi_{d}(q)}
\end{aligned}
$$

where the $\Phi_{d}(q)$ were defined in Section 4.2, Considering the $\Phi_{d}(q)$ as polynomials in $q$ and applying Formula (8), we obtain

$$
\prod_{d \geq 1} \psi_{d}\left(\sum_{\lambda \in \mathcal{P}} r_{\lambda}(q)^{m-1} t^{|\lambda|}\right)^{\Phi_{d}(q)}=\operatorname{Pow}\left(\sum_{\lambda \in \mathcal{P}} r_{\lambda}(q)^{m-1} t^{|\lambda|}, q-1\right) .
$$

For any $d \geq 1$ let $R_{d}^{\text {ind }} \subset R_{d}$ denote the set of absolutely indecomposable representations. This subset is invariant under the action of $G_{d}$ and the quotient can be identified with the set of isomorphism classes of $d$-dimensional absolutely indecomposable representations.

Corollary 5.2. We have

$$
\sum_{d \geq 0}\left|R_{d}^{\mathrm{ind}} / G_{d}\right| t^{d}=(q-1) \log \left(\sum_{\lambda} r_{\lambda}(q)^{m-1} t^{|\lambda|}\right)_{q=|k|}
$$

where the sum ranges over all partitions.

Proof. For any finite field $\mathbb{F}_{q}$, let

$$
M_{d}(q)=\left|R_{d}\left(\mathbb{F}_{q}\right) / G_{d}\left(\mathbb{F}_{q}\right)\right|, \quad A_{d}^{\text {ind }}(q)=\left|R_{d}^{\text {ind }}\left(\mathbb{F}_{q}\right) / G_{d}\left(\mathbb{F}_{q}\right)\right| .
$$

We know from the previous theorem that $M_{d}(q)$ is a polynomial in $q$. One can prove [12, Lemma 5] that $A_{d}^{\text {ind }}(q)$ is also a polynomial in $q$ and

$$
\sum_{d \geq 0} M_{d}(q) t^{d}=\operatorname{Exp}\left(\sum_{d \geq 1} A_{d}^{\text {ind }}(q) t^{d}\right) .
$$

Applying the previous theorem, we obtain $\sum_{d \geq 1} A_{d}^{\text {ind }}(q) t^{d}=$

$$
=\log \operatorname{Pow}\left(\sum_{\lambda} r_{\lambda}(q)^{m-1} t^{|\lambda|}, q-1\right)=(q-1) \log \left(\sum_{\lambda} r_{\lambda}(q)^{m-1} t^{|\lambda|}\right) .
$$




\section{Counting Subgroups}

The aim of this section is to relate the counting polynomials $A_{d}^{\mathrm{irr}}(q)$ with the number of index $d$ subgroups of $\Gamma=F_{m}$. Such a relation is motivated by ideas of $\mathbb{F}_{1}$-geometry [9]: viewing the symmetric group $S_{n}$ as $\mathrm{GL}_{n}$ over a hypothetical field with one element, one can expect counts of permutation representations of a group, or, equivalently, (conjugacy classes of) subgroups of finite index, to arise from the counts of representations over fields with $q$ elements by an appropriate limit process $q \rightarrow 1$. This is accomplished in Lemma 6.4.

6.1. Subgroups and permutation representations. Let $G$ be a finitely generated group. A (permutation) representation of $G$ of order $n$ is an action of $G$ on $[n]=\{1, \ldots, n\}$. It is called irreducible if the action of $G$ is transitive. One can define the notion of an isomorphism between two representations in a natural way. Any irreducible order $n$ representation can be written in the form $G / H$, where $H$ is an index $n$ subgroup of $G$. Two irreducible representations $G / H$ and $G / H^{\prime}$ are isomorphic if and only if $H$ and $H^{\prime}$ are conjugate. Moreover

$$
\operatorname{Aut}(G / H) \simeq N_{G} H / H .
$$

Let $J_{n}$ denote the set of index $n$ subgroups and $I_{n}$ denote the set of conjugacy classes of index $n$ subgroups (or isoclasses of irreducible order $n$ representations). Given a representation $M=G / H$ in $I_{n}$, the number of elements in the conjugacy class of $H$ is equal to

$$
\left|G / N_{G} H\right|=\frac{|G / H|}{\left|N_{G} H / H\right|}=\frac{n}{|\operatorname{Aut} M|} .
$$

Therefore

$$
\frac{\left|J_{n}\right|}{n}=\sum_{M \in I_{n}} \frac{1}{|\operatorname{Aut} M|}
$$

Remark 6.1. We should call an irreducible representation $G / H$ absolutely irreducible if $|\operatorname{Aut}(G / H)|=1$, that is, $N_{G} H=H$. This means that $\left|J_{n}\right| / n$ counts all irreducible representations and not just the absolutely irreducible.

6.2. Two formulas. The set $R_{n}=\operatorname{Hom}\left(G, S_{n}\right)$ is equipped with an action of $S_{n}$ by conjugation. Let $R_{n}^{\text {irr }} \subset R_{n}$ be the set of irreducible representations. Then

$$
I_{n}=R_{n}^{\mathrm{irr}} / S_{n}
$$

According to [16, Ex. 5.13]

$$
\begin{gathered}
\sum_{n \geq 0} \sum_{M \in R_{n} / S_{n}} \frac{t^{n}}{\mid \text { Aut } M \mid}=\sum_{n \geq 0} \frac{\left|R_{n}\right|}{\left|S_{n}\right|} t^{n}=\exp \left(\sum_{n \geq 1} \sum_{M \in I_{n}} \frac{t^{n}}{|\operatorname{Aut} M|}\right), \\
\sum_{n \geq 0}\left|R_{n} / S_{n}\right| t^{n}=\operatorname{Exp}\left(\sum_{n \geq 1}\left|I_{n}\right| t^{n}\right) .
\end{gathered}
$$

The second formula is a standard relation between all representations and indecomposable representations. 
Remark 6.2. The second formula is equivalent to the statement of [16, Ex. 5.13.c], because

$$
\operatorname{Hom}\left(G \times \mathbb{Z}, S_{n}\right)=\left\{(M, g) \in R_{n} \times S_{n} \mid g \in \operatorname{Aut} M\right\}
$$

and therefore

$$
\frac{\left|\operatorname{Hom}\left(G \times \mathbb{Z}, S_{n}\right)\right|}{\left|S_{n}\right|}=\sum_{M \in R_{n}} \frac{\mid \text { Aut } M \mid}{\left|S_{n}\right|}=\left|R_{n} / S_{n}\right| .
$$

Remark 6.3. One can define the "formal" Hall algebra for permutation representations as follows. Let $J=\bigcup_{n \geq 1} I_{n}$ be the set of isoclasses of all irreducible representations. Each $S \in I_{n}$ has two integer parameters $\operatorname{dim} S=n$ and $\mid$ Aut $S \mid$. All representations are parametrized by maps $m: J \rightarrow \mathbb{N}$ with finite support. Note that

$$
\mid \text { Aut } m\left|=\prod_{S \in J} m_{S} ! \cdot\right| \text { Aut }\left.S\right|^{m_{S}},
$$

while in the usual semisimple category $\mid$ Aut $m\left|=\prod_{S \in J}\right| \mathrm{GL}_{m_{S}}($ End $S) \mid$. The "formal" Hall algebra $H$ has these maps as a basis and has multiplication given by

$$
m \circ n=\prod_{S \in J}\left(\begin{array}{c}
m_{S}+n_{S} \\
m_{S}
\end{array}\right) \cdot[m \oplus n] .
$$

There is an integration map $I: H \rightarrow \mathbb{Q} \llbracket t \rrbracket$ (the latter algebra has the usual multiplication) given by

$$
m \mapsto \frac{t^{\operatorname{dim} m}}{|\operatorname{Aut} m|}=\prod_{S \in J} \frac{t^{m_{S} \operatorname{dim} S}}{m_{S} !|\operatorname{Aut} S|^{m_{S}}} .
$$

Formula (11) can be obtained by analyzing this integration map.

6.3. The case $G=F_{m}$. The polynomials $A_{d}^{\mathrm{irr}}(q)$ count absolutely irreducible representations, while $\left|J_{n}\right| / n$ counts all irreducible permutation representations. There is a straightforward relation between these counts:

Lemma 6.4. We have

$$
\Psi\left(\sum_{n \geq 1} \frac{A_{n}^{\mathrm{irr}}(q)}{q-1} t^{n}\right)_{t=\frac{x}{(q-1)^{m-1}}, q=1}=\sum_{n \geq 1} \frac{\left|J_{n}\right|}{n} x^{n} .
$$

Proof. This follows from

$$
\begin{gathered}
\Psi\left(\sum_{n \geq 1} \frac{A_{n}^{\mathrm{irr}}(q)}{1-q} t^{n}\right)=\log \left(T^{-1}\left(\sum_{n \geq 0}[n]_{q}^{!(m-1)}\left((q-1)^{m-1} t\right)^{n}\right)^{-1}\right), \\
\sum_{n \geq 1} \frac{\left|J_{n}\right|}{n} x^{n}=\log \left(\sum_{n \geq 0} n !^{m-1} x^{n}\right) .
\end{gathered}
$$

An explanation for this relation could be that in order to pass from absolutely irreducible representations to all irreducible representations we have to apply Adams operations as in [14, Lemma 3.2]. 


\section{REFERENCES}

[1] Tom Bridgeland, An introduction to motivic Hall algebras, Adv. Math. 229 (2012), no. 1, 102-138, arXiv:1002.4372

[2] Steve Butler, The number of ordered tuples with no global factor, 2006, Preprint.

[3] Samuel Cavazos and Sean Lawton, E-polynomial of $\mathrm{SL}_{2}(\mathbb{C})$-Character Varieties of Free groups, 2014, arXiv:1401.0228

[4] Carlos Florentino and Sean Lawton, The topology of moduli spaces of free group representations, Math. Ann. 345 (2009), no. 2, 453-489.

[5] _ Singularities of free group character varieties, Pacific J. Math. 260 (2012), no. 1, 149-179.

[6] Marshall Hall, Jr., Subgroups of finite index in free groups, Canadian J. Math. 1 (1949), $187-190$.

[7] Tamás Hausel and Fernando Rodriguez-Villegas, Mixed Hodge polynomials of character varieties, Invent. Math. 174 (2008), no. 3, 555-624, arXiv:math/0612668, With an appendix by Nicholas M. Katz.

[8] Jiuzhao Hua, Counting representations of quivers over finite fields, J. Algebra 226 (2000), no. 2, 1011-1033.

[9] Javier López Peña and Oliver Lorscheid, Mapping $\mathbb{F}_{1}$-land: an overview of geometries over the field with one element, Noncommutative geometry, arithmetic, and related topics, 241265, Johns Hopkins Univ. Press, Baltimore, 2011.

[10] Alexander Lubotzky and Dan Segal, Subgroup growth, Progress in Mathematics 212, Birkhäuser, Basel 2003.

[11] I. G. Macdonald, Symmetric functions and Hall polynomials, second ed., Oxford Mathematical Monographs, Oxford University Press, 1995, With contributions by A. Zelevinsky.

[12] Sergey Mozgovoy, A computational criterion for the Kac conjecture, J. Algebra 318 (2007), no. 2, 669-679, arXiv:math/0608321

[13] - Motivic Donaldson-Thomas invariants and McKay correspondence, 2011, arXiv: 1107.6044

[14] Sergey Mozgovoy and Markus Reineke, On the number of stable quiver representations over finite fields, J. Pure Appl. Algebra 213 (2009), no. 4, 430-439, arXiv:0708.1259

[15] C. S. Seshadri, Geometric reductivity over arbitrary base, Advances in Math. 26 (1977), no. 3, 225-274.

[16] Richard P. Stanley, Enumerative combinatorics. Vol. 2, Cambridge Studies in Advanced Mathematics, vol. 62, Cambridge University Press, Cambridge, 1999, With a foreword by Gian-Carlo Rota and appendix 1 by Sergey Fomin.

E-mail address: mozgovoy@maths.tcd.ie

E-mail address: mreineke@uni-wuppertal.de 